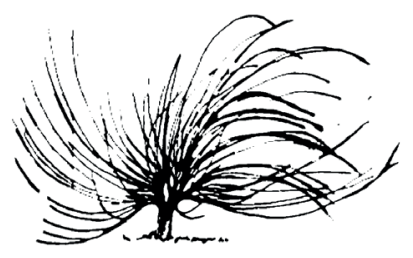

\title{
Estrategias pedagógicas basadas en el enfoque por competencias: una experiencia en el curso de Diplomática
}

Priscilla Carranza Marchena ${ }^{1}$

Universidad Nacional

Costa Rica

priscilla.carranza.marchena@una.ac.cr

Melany Landaverde Recinos ${ }^{2}$

Ministerio de Educación Pública

Costa Rica

mlandaverde822@gmail.com

\begin{abstract}
Resumen
Este artículo presenta los resultados de una investigación realizada en el curso de Diplomática, perteneciente al Bachillerato en Archivística, de la Universidad de Costa Rica (UCR), y su relación con el enfoque por competencias establecido en la reforma curricular vigente de este programa de estudio. Para llevar a cabo esta indagación, se aplicaron tres instrumentos: una entrevista a la docente del curso, un cuestionario dirigido al estudiantado del curso de Diplomática y una observación de clase cuyo objetivo principal fue obtener información basada en las estrategias pedagógicas utilizadas en el curso y su congruencia con el enfoque por competencias. Asimismo, se generó una producción didáctica (manual) basada en estrategias pedagógicas
\end{abstract}

Recibido: 5 de abril de 2019. Aprobado: 28 de octubre de 2019.

http://dx.doi.org/10.15359/rep.15-1.8

1 Académica, Universidad Nacional. Magíster en Educación con énfasis en Docencia Universitaria, Universidad Nacional.

2 Profesional en archivística, Ministerio de Educación Pública (MEP). Magíster en Educación con énfasis en Docencia Universitaria, Universidad Nacional. 
bajo el enfoque por competencias, la cual se socializó mediante un taller y se recolectaron datos en cuanto a la pertinencia de la propuesta pedagógica planteada. Los resultados obtenidos evidenciaron que, en el curso hay estrategias que podrían ser mejoradas e incorporadas. Se concluyó que el manual generado es un producto único en su índole, en el Bachillerato en Archivística de la Universidad de Costa Rica (UCR), el cual puede ser un recurso de utilidad y enriquecimiento para el proceso de mediación pedagógica.

Palabras clave: estrategias pedagógicas, enfoque por competencias, Diplomática.

\begin{abstract}
This article presents the results of a research carried out in the Diplomatics course, which belongs to the Bachelor's Program in Archive of the University of Costa Rica (UCR) and its connection with the Competency-Based Learning Approach established in the current syllabus of this bachelor's degree. To carry out this research, three instruments were applied: an interview to the professor of the course, a questionnaire to the students taking the Diplomatics course, and a class observation guide. These instruments aimed to gather information based on the teaching strategies implemented in the course and their relationship with the competency-based approach. In addition, a manual was created and socialized in a workshop, where data related to the presented proposal was gathered. The obtained results showed that there are some teaching strategies that could be improved and others that should be included. It was concluded that the manual created is a unique product for this major since it can be a useful and enriching resource for the mediation process of the Diplomatics course and for the Bachelor's Program in Archive.
\end{abstract}

Keywords: teaching strategies, competency-based learning approach, Diplomatics course. 


\section{Introducción}

$\mathrm{E}$

proceso de enseñanza y aprendizaje es complejo; como tal, se deben adoptar, cambiar o modificar las prácticas pedagógicas para alcanzar objetivos académicos de manera óptima y efectiva. Este artículo presenta la investigación desarrollada como respuesta a la aprobación de la reforma curricular bajo el enfoque por competencias del Bachillerato en Archivística, perteneciente a la Escuela de Historia, de la Universidad de Costa Rica (UCR). La indagación se orientó en las estrategias pedagógicas basadas en el enfoque por competencias en el curso de Diplomática; este estudio pretendía impactar dicho curso, específicamente, mediante la recolección informativa relacionada con las estrategias pedagógicas transversales y específicas utilizadas, con respecto a la reforma curricular vigente.

El planteamiento por competencias se fundamenta en la propia capacidad y responsabilidad del estudiantado, así como en el desarrollo de su autonomía, renovando, así, el sistema antiguo centrado exclusivamente en el profesorado. Asimismo, García (2011) afirma que este enfoque consiste en desarrollar las competencias transversales (instrumentales, interpersonales y sistémicas) necesarias y las específicas (propias de cada profesión), con el propósito de capacitar a la persona en cuanto a conocimientos científicos y técnicos aplicables en contextos diversos y complejos, integrando sus propias actitudes y valores de forma personal y profesional.

El Bachillerato en Archivística de la Universidad de Costa Rica (UCR), en particular, establece en su plan de estudio (2011) el enfoque por competencias. Este pretende que el alumnado desarrolle competencias instrumentales tales como capacidad de análisis y síntesis; capacidad de organización; planificación y gestión de la información; comunicación oral y escrita en la lengua nativa; resolución de problemas y toma de decisiones. Además, tiene como objetivo que el alumnado se desenvuelva interpersonalmente, a través del trabajo en equipo, del razonamiento crítico y del compromiso ético. Finalmente, este plan de estudio (2011) procura fomentar competencias sistemáticas como el aprendizaje autónomo, la adaptación a situaciones nuevas, la creatividad, el liderazgo, la iniciativa con espíritu emprendedor y la motivación por la calidad. 
En este sentido, las estrategias de enseñanza deben ser diseñadas de manera que estimulen la observación, el análisis, la generación de opiniones, la formulación de hipótesis, la búsqueda de soluciones y el descubrimiento del conocimiento. Por lo tanto, es fundamental mediar las clases con ambientes de aprendizaje desafiantes pero agradables, que promuevan el aprendizaje; también, es esencial y tarea del docente lograr que el estudiantado sea responsable de su propio aprendizaje.

\section{Referente teórico}

\section{El currículum bajo el enfoque por competencias}

Recientemente, se han generado cambios en el nivel del sistema productivo, producto de los procesos de globalización y, específicamente, generados a partir de las transformaciones que se han generado y se están gestando en el campo laboral, los cuales inciden y afectan directamente tanto la construcción como la organización de los sistemas educativos. Asimismo, debido a una serie de demandas e intereses de diferente índole (del sector político, de los directivos y de autoridades educativas, intelectuales y docentes), planteados socialmente en diferentes épocas, escenarios y momentos históricos, es que se han instaurado desafíos determinados en el ámbito educativo.

En esta perspectiva, Tobón, Pimienta y García (2010) señalan que "las demandas se convierten en retos cuando se analizan, se comprenden, se discuten y se acuerdan ejes mínimos hacia los cuales orientar los procesos educativos" (p. 2), lo que conlleva tomar acuerdos que deben guiar y contextualizar la educación de forma óptima. Cuando la formación responde a las necesidades de uno u otro grupo, ya sean implícitas o explícitas, se crea un paradigma educativo en beneficio de uno o varios, pero desconociendo, en ocasiones, los intereses de una comunidad educativa y su contexto particular. En este sentido, es válido cuestionarse ¿qué es un paradigma? Tobón, Pimienta y García (2010) se refieren a este término como el "conjunto de creencias, valores y argumentos compartidos por una comunidad científica en un tiempo determinado" (p. 2).

En la línea aludida, podría establecerse que el modelo por competencias es un nuevo paradigma, ya que surgió en la educación como una alternativa para trabajar otras teorías de aprendizaje tradicionales (conductismo, cognoscitivismo y constructivismo), cuyos planteamientos 
en el nivel teórico y metodológico, no siempre, ofrecen opciones viables para abordar los cambios actuales, debido a que las necesidades y tendencias sociales varían de generación en generación y puesto que la nueva perspectiva educativa implica el tránsito de la lógica de los contenidos a la de la acción. Para Luengo, Luzón y Torres (2008), el concepto de competencia surgió en la literatura anglosajona en los noventa; este favoreció el apogeo de la psicología diferencial o cognitiva. Sin embargo, esta perspectiva recibió críticas en contra, dado su énfasis en la individualidad y la carencia de una dimensión colectiva o social. De manera posterior, se gestó un nuevo enfoque basado en la sociología francófona, el cual enfatizaba la parte social de la competencia y explicaba cómo esta se adquiere, reconoce y aplica.

Asimismo, cuando se trabaja bajo el enfoque por competencias, Posada (2004, p. 26) recomienda tomar en cuenta los cuatro pilares de la educación:

a. Aprender a conocer: concertar entre una cultura general suficientemente amplia y los conocimientos particulares de las diferentes disciplinas, en torno a problemas e interrogantes concretos. Esto requiere aprender a aprender, con el fin de aprovechar las posibilidades que ofrece la educación a lo largo de la vida.

b. Aprender a hacer: adquirir no sólo una certificación profesional, sino más bien competencias que capaciten al individuo para hacer frente a gran número de situaciones previstas e imprevistas y a trabajar en equipo.

c. Aprender a vivir juntos: realizar proyectos comunes y prepararse para asumir y resolver los conflictos, respetando los valores del pluralismo, el entendimiento mutuo y la paz, a través de la comprensión del otro y de las formas de interdependencia.

d. Aprender a ser: actuar con creciente capacidad de autonomía, de juicio y responsabilidad personal, para que florezca en mejor forma la propia personalidad. Con tal fin, no subestimar ninguna posibilidad de cada individuo en su proceso educativo: competencias intelectuales (memorizar, razonar, comprender, etc.), comunicativas, afectivas, estéticas, físicas, entre otras.

Además, cabe destacar lo que realmente ofrece el enfoque por competencias que le permite posicionarse como el nuevo paradigma 
educativo. Tobón, Pimienta y García (2010, p. 4) indican que son variados los problemas a los que responde, en forma clara y consistente, este modelo, ya que enfatiza en:

a. Cómo gestionar el currículo y el microcurrículo para asegurar la calidad del aprendizaje en un marco sistémico.

b. Cómo lograr que el currículo y los procesos de aprendizaje y evaluación sean pertinentes para los y las estudiantes y las dinámicas del contexto local, nacional e internacional, actual y futuro.

c. Cómo formar personas para afrontar los problemas cotidianos integrando y movilizando el saber ser, el saber hacer y el saber conocer, al considerar los retos del contexto.

d. Qué es la idoneidad y cómo se inserta en la educación dentro de los procesos de enseñanza y aprendizaje.

e. Cómo formar personas con habilidades críticas, reflexivas, analíticas y creativas, que las apliquen realmente en la vida cotidiana (p. 4).

Las respuestas a los problemas planteados previamente consideran los cambios que se generan en los contextos social, laboral-profesional, científico, etc., lo que le permite al enfoque por competencias consolidarse cada día, mucho más, como el nuevo paradigma educativo; prueba de esto es su progresiva aceptación entre las entidades internacionales, múltiples instituciones educativas y académicos en pedagogía. No obstante, es válido aclarar que, cuando se analizan las principales fallas de la educación tradicional, con su lógica centrada en los contenidos, se descubre que aquellas se relacionan con la carencia en la aplicación de los saberes, la falta de pertinencia de los propósitos educativos y el excesivo énfasis en lo académico. El enfoque por competencias, por lo contrario, apoya el acercamiento y entrelazamiento de las instituciones educativas con la sociedad y sus dinámicas de cambio, con el fin de que el estudiantado y futuro profesional estén en condiciones de contribuir tanto al desarrollo social y económico como al equilibrio ambiental y ecológico.

Por consiguiente, se está ante un modelo que busca satisfacer las grandes necesidades de la educación actual y que trata, a su vez, de generar prácticas docentes más acordes con los nuevos cambios sociales. El modelo por competencias, como nuevo paradigma educativo, y 
según Tobón, Pimienta y García (2010, p. 6), tiene una serie de principios en los que existe cada vez mayor consenso, entre ellos están:

a. Pertinencia: Las instituciones educativas deben generar sus propuestas de formación articulando su visión y filosofía con los retos del contexto y las políticas educativas vigentes.

b. Calidad: Los procesos educativos deben asegurar la calidad del aprendizaje en correspondencia con un determinado perfil de formación, considerando la participación de la comunidad.

c. Formar competencias: Los docentes deben orientar sus acciones a formar competencias y no a enseñar contenidos, los cuales deben ser sólo medios.

d. Papel del docente: Deben ser ante todo guías, dinamizadores y mediadores, para que los y las estudiantes aprendan y refuercen las competencias. No deben ser sólo transmisores de contenidos.

e. Generación del cambio: El cambio educativo se genera mediante la reflexión y la formación de directivos, maestras y maestros. No se genera en las políticas ni en las reformas del currículo.

f. Esencia de las competencias: Las competencias son actuaciones o desempeños ante actividades y situaciones cotidianas que articulan y movilizan recursos personales y del contexto externo.

g. Componentes de una competencia: Lo más acordado es que una competencia se compone de conocimientos, habilidades y actitudes en forma articulada.

Dichos principios deben seguirse en su interpretación, argumentación y presentación, a través de propuestas educativas viables que conduzcan a una práctica docente congruente con el modelo pedagógico o andragógico establecido y que permitan la constante capacitación para la comprensión y el análisis de la información relativa a los contextos educativos. Por ende, el desarrollo de competencias en el enfoque por competencias en el alumnado del Bachillerato en Archivística de la Universidad de Costa Rica (UCR) implica un desafío, ya que este enfoque trasciende los conocimientos conceptuales hacia un saber hacer y saber ser, siguiendo un modelo compuesto por competencias transversales y específicas. 


\section{Curso de Diplomática del plan de estudio del Bachillerato en Archivística de la Universidad de Costa Rica (UCR)}

En cuanto al diseño curricular del curso de Diplomática, este, claramente, señala la definición de áreas curriculares divididas en las siguientes categorías: Área de Teoría y Métodos, Área de Tratamiento y Servicios Archivísticos, Área de Administración y Derecho y Área de Tecnologías de la Información y Comunicación. Cada una de estas presenta una breve descripción sobre su abordaje y propósito en el curso. Asimismo, el programa del curso hace referencia a la formación integral, a través del conocimiento transversal sobre las tecnologías de la información (TIC), el tratamiento archivístico, la ética profesional, la calidad en la gestión de documentos, la formación teórico-práctica contextualizada y el balance entre los sistemas públicos y privados. También, se puntualiza en el diseño curricular por competencias, en particular, las instrumentales (análisis y síntesis), interpersonales (razonamiento crítico), sistémicas (creatividad e innovación) y teóricas y metodológicas (técnicas de trabajo confiables y válidas). De acuerdo con lo mencionado, el curso tiene como objetivo principal la formación integral para que el estudiantado sea capaz de desempeñarse en el mercado laboral de forma atinente y así se adapte a posibles cambios o nuevas tendencias.

\section{Perfil docente por competencias}

La formación basada en competencias está en el centro de una serie de transformaciones educativas, lo que requiere profundos cambios y nuevos perfiles tanto estudiantiles como docentes. El rol del profesorado se compromete a realizar modificaciones con respecto a las actividades y estrategias; este proceso debe ser incorporado al planeamiento, como base o guía para la consecución de objetivos, conocimientos y competencias, de modo tal que el aprendizaje sea funcional para enfrentar diversos desafíos académicos y profesionales. El papel docente debe ser el de un agente de cambio en el que se exige un compromiso personal y de reflexión constante. Álvarez (2011, p. 102) señala que esto implica nuevas funciones, tales como:

a. Preparar a los y las estudiantes para que se adapten a la cultura vigente y para el futuro. 
b. Acompañar, orientar y guiar el trabajo y la búsqueda del estudiante.

c. Promover el desarrollo integral y el mejoramiento continuo del estudiante.

d. Apoyar y sostener el esfuerzo irrenunciable del estudiante.

e. Diseñar escenarios, procesos y experiencias de aprendizaje significativas y relevantes.

En este sentido, según Jaén (2011), para que el nuevo plan de estudio bajo el enfoque por competencias del Bachillerato en Archivística de la Universidad de Costa Rica (UCR) funcione óptimamente, es indispensable que el profesorado reúna las siguientes características: grado académico de máster o de doctor, preferiblemente con estudios en el exterior; especialidad en algunas áreas, si son profesores de otras áreas, deben tener conocimiento en archivística; experiencia profesional en archivos y en investigación; producción bibliográfica; dominio de idiomas, y disponibilidad para actualizarse constantemente.

\section{Estrategias pedagógicas bajo el enfoque por competencias}

Las estrategias pedagógicas pueden definirse como las acciones educativas previamente organizadas que se utilizan durante el proceso de enseñanza y aprendizaje. Estas implican alcanzar la comprensión de contenidos y lograr objetivos específicos preestablecidos e involucran procesos cognitivos, procedimientos y ejercicios didácticos propios de la formación. En el contexto educativo actual, se dan múltiples interpretaciones o formas de comprender, planificar y aplicar el concepto de estrategia; desde esta perspectiva, es importante señalar que una estrategia es diferente a una actividad, método o metodología de enseñanza. Por tal razón, se clarifica que en una estrategia no hay cabida para la improvisación, arbitrariedad o casualidad; por lo contrario, para que aquella funcione, es esencial tanto la organización como la planificación intencional y determinada hacia un objetivo en particular. Asimismo, una estrategia consiste en un conjunto de acciones procedimentales visiblemente planeadas con las cuales se pretende alcanzar una finalidad; una estrategia debe ser repetible y, a través de su utilización, en condiciones y circunstancias parecidas, proveer resultados similares.

De acuerdo con Camacho, Flórez, Gaibao, Aguirre, Castellanos y Murcia (2012, p. 6), las estrategias pedagógicas se dividen en: 
a. Estrategias cognitivas: permiten desarrollar una serie de acciones encaminadas al aprendizaje significativo de las temáticas en estudio.

b. Estrategias metacognitivas: conducen al estudiante a realizar ejercicios de conciencia del propio saber, a cuestionar lo que se aprende, cómo se aprende, con qué se aprende y su función social.

c. Estrategias lúdicas: facilitan el aprendizaje mediante la interacción agradable, emocional y la aplicación del juego.

d. Estrategias tecnológicas: hoy, en todo proceso de aprendizaje el dominio y aplicación de las tecnologías, hacen competente a cualquier tipo de estudiante.

e. Estrategias socio-afectivas: propician un ambiente agradable de aprendizaje.

En el curso de Diplomática, el propósito principal de las estrategias pedagógicas es dirigir el proceso de aprendizaje significativamente, lo cual se alcanza por medio de la interacción y de un ambiente propicio, aspectos que, unificados, configuran una experiencia cognitiva efectiva. Por otra parte, el enfoque por competencias es un modelo que plantea la utilización de estrategias pedagógicas. Entre los representantes de los sectores industriales, gubernamentales y educativos, este tipo de educación ha generado controversia, pero también se ha considerado un buen inicio para elevar los niveles competitivos y aumentar los recursos que se invierten en programas de capacitación.

Desde esta óptica, debe existir relación entre la educación superior y el trabajo, lo que requiere una formación profesional basada en competencias, no solo las relacionadas con el ámbito laboral, sino también comunicativas, intelectuales y socioafectivas que promuevan un desempeño adecuado en los complejos campos académicos y profesionales. El conocimiento propiamente en la acción contribuye a evitar prácticas pedagógicas academicistas, en las cuales la teoría y los conocimientos se asumen como fines en sí mismos, con poca o nula aplicación práctica. Por ello, Posada (2004, p. 12) afirma que las estrategias pedagógicas deben tener siempre presente el desempeño del estudiantado, lo que implica: 
a. Destacar la relevancia y pertinencia de los contenidos que se aprenden.

b. Evitar el fraccionamiento tradicional de los conocimientos y facilitar su integración.

c. Generar aprendizajes aplicables a situaciones complejas.

d. Estimular, facilitar y provocar la autonomía personal del aprendiz.

Asimismo, Posada (2004) destacó que la educación basada en competencias requiere modificarse en el nivel curricular, en cuanto al rol docente y dicente, $\mathrm{y}$ en relación con las estrategias pedagógicas utilizadas; en tal sentido, sus postulados (2004, pp. 12-13) proponen:

a. Competencias identificables, verificables y de conocimiento público. Cuando el estudiante sabe lo que se espera de él se muestra más eficiente y motivado.

b. Instrucción dirigida al desarrollo y evaluación individual de cada competencia.

c. Evaluación teniendo en cuenta el conocimiento, las habilidades, las actitudes y el desempeño como principales fuentes de evidencia.

d. Progreso de los estudiantes al ritmo de cada uno.

e. Instrucción individualizada al máximo posible.

f. Énfasis puesto en los logros.

g. Participación de los estudiantes en la elaboración de las estrategias de aprendizaje.

h. Experiencias de aprendizaje guiadas por una permanente retroalimentación.

i. Amplia variedad de materiales didácticos, con orientación del aprendizaje hacia la solución de problemas, más que a la repetición de contenidos.

j. Programas modulares y abiertos que faciliten el movimiento y la reincorporación de los estudiantes en diferentes momentos.

El enfoque por competencias enfatiza la actitud del estudiantado, que deja de ser pasivo y receptor para convertirse en creativo, activo, protagonista, autónomo, crítico, reflexivo, capaz de resolver problemas y con ansias de superación. El aprendizaje se hace desde las propias cultura y experiencia a lo largo de toda la vida. 


\section{Metodología}

\section{Enfoque y tipo de estudio}

Esta investigación fue un estudio de caso enfocado específicamente en el curso de Diplomática, perteneciente al Bachillerato en Archivística de la Universidad de Costa Rica (UCR). Se desarrolló bajo el paradigma cualitativo, mediante el cual, según Monje (2011), los “investigadores se aproximan a un sujeto real, un individuo real, que está presente en el mundo y que puede, en cierta medida, ofrecernos información sobre sus propias experiencias, opiniones, valores etc." (p. 32).

El enfoque exploratorio fue utilizado; de acuerdo con Hernández, Fernández y Baptista (2010):

Los estudios exploratorios se realizan cuando el objetivo es examinar un tema o problema de investigación poco estudiado, del cual se tienen muchas dudas o no se ha abordado antes. Es decir, cuando la revisión de la literatura reveló que tan sólo hay guías no investigadas e ideas vagamente relacionadas con el problema de estudio, o bien, si deseamos indagar sobre temas y áreas desde nuevas perspectivas (p. 79).

En este sentido, en Costa Rica, el campo de la archivística, con respecto al proceso de enseñanza y aprendizaje en educación superior, en particular, no ha sido profundamente investigado hasta la fecha. De este modo, el análisis se realizó con base en tres categorías de análisis: las estrategias pedagógicas utilizadas durante el proceso de enseñanza del curso de Diplomática, las estrategias pedagógicas basadas en el enfoque por competencias y la percepción de los participantes con respecto a la pertinencia de las estrategias pedagógicas basadas en el enfoque por competencias planteadas en la producción didáctica generada (manual). Esta información fue usada para documentar una situación real vivida en el espacio educativo seleccionado y así fortalecer su proceso de enseñanza actual.

Monje (2011) establece que la investigación cualitativa tiene como propósito principal la observación de un mundo social, los eventos, situaciones y experiencias de este, lo cual implica recabar y registrar información, sistemáticamente, de los hechos ocurridos en el transcurso de la indagación, para así alcanzar los objetivos establecidos con precisión. Por ende, este trabajo se enfocó en el estudio de la realidad del 
curso de Diplomática y de sus participantes, para comprender, de forma detallada, las vivencias de ese escenario educativo y social, en términos del enfoque por competencias y de las estrategias pedagógicas utilizadas en el proceso de enseñanza.

\section{Población de estudio}

Esta investigación se llevó a cabo en la Universidad de Costa Rica, específicamente, con el estudiantado del curso de Diplomática, perteneciente al Bachillerato en Archivística de esta universidad estatal costarricense. El curso pertenece al II ciclo (modalidad semestral; naturaleza teórico-práctica) del primer año del bachillerato y tiene como propósito principal desarrollar el análisis crítico de la Diplomática, con respecto a la génesis y a la conservación de documentos contemporáneos. Asimismo, este curso pretende desarrollar competencias en cuanto a la diplomática (como ciencia auxiliar de la historia), los tipos de archivo y las tipologías documentales; por esa razón, se enfoca en el análisis, la síntesis, el razonamiento crítico, la creatividad, la innovación y la implementación tanto de métodos como de técnicas legítimas y auténticas. La muestra estuvo conformada por diez discentes, cuyo rango de edad comprendió entre los dieciocho y los treinta años; la académica a cargo del curso, y nueve profesores, quienes laboran para esta carrera en particular y fueron parte del proceso de socialización y el taller, en el cual se recabaron datos acerca de su percepción en relación con las estrategias pedagógicas propuestas y su pertinencia con respecto al enfoque por competencias.

\section{Instrumentos}

Para el proceso de triangulación y análisis de la información, se recolectaron datos de tres instrumentos. El primero corresponde a una entrevista cuyo propósito principal fue recabar información basada en el proceso de enseñanza del curso de Diplomática, específicamente, en relación con las estrategias pedagógicas implementadas y su correspondencia con el enfoque por competencias. Esta herramienta fue aplicada a la académica a cargo del curso de Diplomática e incluía interrogantes tales como ¿qué comprende por competencias?, ¿cómo estas nuevas nociones sobre competencias están siendo aplicadas para el desarrollo de las estrategias pedagógicas?, ¿cuáles estrategias pedagógicas utiliza?, ¿qué recurso material, físico, humano o tecnológico utiliza para el desarrollo de las estrategias pedagógicas?, entre otras. 
Asimismo, se aplicó un cuestionario, con preguntas abiertas y cerradas, dirigido al estudiantado del curso; este, con el objetivo de recolectar información fundamentada en las estrategias pedagógicas utilizadas durante las lecciones de Diplomática. Finalmente, con base en la información recabada por medio de los instrumentos previamente descritos, se generó la producción didáctica (manual) y se recopilaron datos acerca de la percepción y valoración por parte del profesorado del Bachillerato en Archivística; este proceso se llevó a cabo mediante un cuestionario de preguntas abiertas.

\section{Categorías de análisis}

Esta investigación se desarrolló con el objetivo principal de fortalecer el proceso de enseñanza del curso de Diplomática del Bachillerato en Archivística de la Universidad de Costa Rica (UCR), de acuerdo con la reforma curricular vigente. Para ello, se establecieron dos categorías de análisis: a) estrategias pedagógicas utilizadas en el curso de Diplomática y b) percepción de los participantes con respecto a la pertinencia de las estrategias pedagógicas basadas en el enfoque por competencias, planteadas en la producción didáctica generada (manual).

\section{Producción didáctica y socialización}

La producción didáctica fue elaborada por las investigadoras, tiene un formato informativo bajo el nombre de Manual de Estrategias Pedagógicas bajo el Enfoque por Competencias. Se generó con base en estrategias pedagógicas de este enfoque e incluye ejercicios reflexivos, información con respecto al currículum por competencias, propuestas para rúbricas de evaluación, el diseño curricular del plan de estudios del Bachillerato en Archivística de la Universidad de Costa Rica, ejercicios prácticos y una sección de consideraciones finales. El objetivo primordial del producto es funcionar como un recurso de apoyo didáctico para fortalecer el proceso de enseñanza del curso de Diplomática, en congruencia con el planteamiento de la reforma curricular vigente. Por lo tanto, el manual se enfocó en atender la necesidad visualizada, en cuanto a las estrategias pedagógicas y las competencias (transversales y específicas); está fundamentado en ejercicios autorreflexivos que permiten la construcción del conocimiento mediante la lectura, el análisis crítico y la discusión. Es esencial mencionar que este escrito se constituye como una herramienta didáctica funcional para el curso de 
Diplomática y el Bachillerato en Archivística, ya que es un producto único en su categoría y, dado que su formato, en general, es sencillo, claro, así como las instrucciones son sugerentes, fue percibido y valorado como un recurso útil y significativo para dicho curso.

La producción didáctica de esta investigación (manual) fue presentada y socializada en la sede Rodrigo Facio de la Universidad de Costa Rica (UCR), en la Escuela de Historia, perteneciente a la Facultad de Ciencias Sociales, durante una reunión de académicos. Este proceso de socialización dio a conocer el trabajo realizado, mediante una explicación detallada y profunda de la investigación completada. Además, se hizo un taller en el cual se aplicaron dos actividades de lectura, análisis, discusión y reflexión, con respecto a las estrategias pedagógicas y las competencias transversales (comunicación oral y escrita de forma efectiva, capacidad de observación, análisis y síntesis, resolución de problemas en diferentes escenarios, trabajo en equipos interdisciplinarios, actuación ética en el desempeño profesional, entre otras) y específicas (dominio de la epistemología archivística, diagnóstico de problemas archivísticos para el planteamiento de soluciones, implementación de métodos y técnicas de investigación válidas y confiables, investigación sobre temas archivísticos para la generación de nuevo conocimiento, organización y dirección de archivos en instituciones públicas y privadas, entre otras) planteadas en la producción didáctica generada. Este proceso fue fundamental para recabar datos acerca de la percepción de los académicos participantes, en relación con las estrategias pedagógicas propuestas y su pertinencia con respecto al enfoque por competencias.

\section{Resultados y discusión}

Los datos recabados mostraron aspectos relevantes con respecto a las estrategias pedagógicas implementadas durante la mediación pedagógica del curso de Diplomática. Estas se enfocan en el modelo de enseñanza que se centra en el profesorado y están fuertemente arraigadas en la comprobación de conocimientos sobre el material escrito (textos, lecturas, entre otros); el proceso se realiza mediante prácticas estructuradas que se relacionan con diferentes casos. Asimismo, la entrevista aplicada a la docente del curso brindó información clave en cuanto al enfoque por competencias y su funcionamiento en el curso de Diplomática. La profesora afirmó que este enfoque se refiere a las aptitudes 
y actitudes que una persona debe desarrollar para desempeñarse eficientemente en su trabajo; por lo tanto, su desenvolvimiento se asocia directamente con la construcción de valores y conocimiento, la creatividad (no con recetas aprendidas), el cambio constante, la adaptabilidad, entre otros. Además, la información compilada en el cuestionario que se aplicó al estudiantado del curso de Diplomática mostró que las estrategias pedagógicas utilizadas en la clase, frecuentemente, se basan en el análisis de lecturas y textos; por lo tanto, los dicentes consideran oportuno y necesario el empleo de estrategias pedagógicas en las cuales se promueva la construcción activa del conocimiento.

Con base en la información previamente recabada, las investigadoras procedieron a la generación de la producción didáctica (manual), la cual fue socializada y trabajada a través de un taller llevado a cabo con el profesorado del Bachillerato en Archivística de la Universidad de Costa Rica (UCR). El Manual de Estrategias Pedagógicas bajo el Enfoque por Competencias fue diseñado con base en estrategias pedagógicas bajo enfoque y su rol principal en la investigación fue el de recurso didáctico de apoyo para el curso de Diplomática. De hecho, la información recolectada arrojó datos importantes en relación con la pertinencia de las estrategias pedagógicas basadas en el enfoque por competencias planteadas. En cuanto a la definición de estrategias pedagógicas, el profesorado señaló que es clara y precisa y que les brindó la posibilidad de comprender de una forma mucho más efectiva el significado de este término. Asimismo, los participantes indicaron que es muy enriquecedor conocer el significado del concepto como tal, los tipos de estrategias pedagógicas existentes y su respectiva clasificación, ya que esa información permite realizar un análisis comparativo e interiorizar los elementos que las componen, así como puntualizar en el/los tipo/s de estrategia/s pedagógica/s que se desea/n utilizar durante una clase o periodo específico.

Además, el profesorado argumentó que la definición de enfoque por competencias es clara y concreta; por lo tanto, se visualiza la relación entre el saber ser, saber hacer y saber conocer como principio fundamental de este enfoque. Cabe mencionar que la definición planteada en el manual abarca ampliamente los aspectos claves vinculados con los pilares del plan de estudio del Bachillerato en Archivística de la Universidad de Costa Rica (UCR). Los académicos mencionaron que existe pertinencia y congruencia entre las estrategias pedagógicas propuestas 
en el manual y el enfoque por competencias, dado que las tablas presentadas plantean claramente gran cantidad y diversidad de estrategias pedagógicas según cada competencia transversal y específica, lo que permite visualizar, de forma coherente y organizada, la información.

También, el profesorado resaltó la importancia del manual como aporte para el Bachillerato en Archivística de la Universidad de Costa Rica (UCR), ya que tenía el conocimiento de las competencias establecidas en la reforma curricular, pero no había total claridad en cómo implementarlas o a través de cuál medio hacerlo de forma específica. En este sentido, la relación entre cada competencia y estrategia/s pedagógica/s planteada/s permitió tener una visión organizada de cómo ponerlas en marcha durante su quehacer pedagógico, al igual que cómo recabar información y sistematizarla, para recopilar datos que promuevan reflexionar y hacer modificaciones a su mediación docente.

El profesorado sugirió que en el manual de estrategias pedagógicas bajo el enfoque por competencias es relevante incorporar estrategias como la mesa redonda, el foro y el debate, con el objetivo de desarrollar clases participativas en las cuales se promueva el análisis y la discusión grupal. Finalmente, los asistentes a la socialización señalaron que el manual presentado es muy completo y único en su categoría, que brinda instrucciones claras y de fácil compresión y que el diseño es atractivo y con colores llamativos. La figura 1 muestra, de forma gráfica, los datos recolectados.

Percepción del manual: Estrategias pedagógicas basadas en el enfoque por competencias

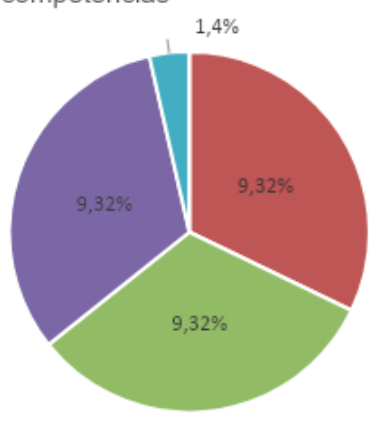

Percepción: Estrategías pedagógicas basadas - en el enfoque por competencias planteadas en el manual

Claridad y precisión en cuanto a la definición de

estrategias pedagógicas

Claridad y precisión en cuanto a la definición del

enfoque por competencias

Pertinencia de las estrategias pedagógicas

- planteadas con respecto al enfoque por competencias

Sugerencias de otras estrategias pedagógicas

bajo el enfoque por competencias

Sugerencias con respecto al contenido y/o

diseño

Figura 1. Percepción de los participantes con respecto a la pertinencia de las estrategias pedagógicas basadas en el enfoque por competencias planteadas en la producción didáctica (manual)

Nota: Elaboración propia de las autoras. 
Como resultado, todo el profesorado (un total de nueve) demostró comprensión analítica con base en la pertinencia de las estrategias pedagógicas bajo en el enfoque por competencias planteadas en el manual. De esta forma, se evidenció el aporte y la utilidad del escrito para el cuerpo docente del Bachillerato en Archivística de la Universidad de Costa Rica (UCR) participante; esto en congruencia con la reforma curricular vigente. Asimismo, los académicos participantes señalaron la importancia de la producción didáctica como recurso de apoyo innovador para el curso de Diplomática.

\section{Conclusiones}

A través de esta investigación, se lograron identificar concretamente las estrategias pedagógicas utilizadas en el curso de Diplomática perteneciente al Bachillerato en Archivística de la Universidad de Costa Rica (UCR). Con base en esos datos, se realizó una búsqueda exhaustiva para el reconocimiento de nuevas tendencias en estrategias pedagógicas fundamentadas en el enfoque por competencias, lo cual brindó la argumentación teórica dirigida al diseño de la producción didáctica (manual) generada. Esta ofrece una amplia gama de estrategias pedagógicas bajo este enfoque, la cual se validó y posteriormente se socializó con el profesorado participante de la Sección de Archivística; así, se recolectaron datos con respecto a su percepción del manual. Como conclusión principal, se establece el fortalecimiento del proceso de enseñanza del curso de Diplomática del Bachillerato en Archivística de la Universidad de Costa Rica (UCR), en congruencia con la reforma curricular vigente y mediante la producción de un manual de estrategias pedagógicas bajo el enfoque por competencias, específicamente, creado como recurso didáctico de apoyo y consulta para este contexto educativo en particular.

De acuerdo con la información recabada con respecto al manual generado, el análisis realizado y la triangulación de datos, se destaca la relevancia, la funcionalidad y el aporte sustancial de la producción didáctica, lo que evidencia su excelencia y calidad como herramienta pedagógica de apoyo para el curso de Diplomática y como recurso de posible consulta para el Bachillerato en Archivística de la Universidad de Costa Rica (UCR). Esto, por ser la primera producción didáctica, en su categoría, que se enfoca en fortalecer el proceso de enseñanza de un curso específico de la carrera y que puede ser una herramienta de soporte y consulta para otros cursos de ese bachillerato. 
Asimismo, esta investigación tuvo un alcance a gran escala en la Sección de Archivística de la Universidad de Costa Rica (UCR), ya que trascendió tanto para la docente del curso de Diplomática como para el profesorado participante que labora para el Bachillerato en Archivística de dicha universidad. Mediante el proceso de socialización del manual de estrategias pedagógicas bajo el enfoque por competencias, se trabajó en la reflexión e importancia de implementar los aspectos propuestos en la producción didáctica generada. Según información suministrada por la Sección de Archivística, no existe registro de material o producciones didácticas como la elaborada, hasta el momento de esta investigación, lo que convierte el manual de estrategias pedagógicas bajo el enfoque por competencias en el único recurso de esta índole de ese bachillerato.

Cabe resaltar que este estudio también tuvo como alcance importante la generación de un espacio para reflexionar acerca de la importancia de tener conocimiento andragógico, filosófico y pedagógico, además de los poseídos en materia archivística. En consecuencia, se puede concluir que se impactó de forma positiva y se fortaleció el proceso de enseñanza del curso de Diplomática del Bachillerato en Archivística de la Universidad de Costa Rica (UCR), de acuerdo con la reforma curricular vigente.

\section{Referencias}

Álvarez, M. (2011). Perfil del docente en el enfoque basado en competencias. Revista Electrónica Educare, XV(1), 99-107. Recuperado de http://www.redalyc.org/articulo.oa?id=194118804008

Camacho, T.; Flórez, M.; Gaibao, D.; Aguirre, M.; Castellanos, Y. y Murcia, G. (2012). Estrategias pedagógicas en el ámbito educativo. Recuperado de https://docplayer.es/13194924-Estrategias-pedagogicas-en-el-ambito-educativo.html

García, J. (2011). Modelo educativo basado en competencias: importancia y necesidad. Revista Actualidades Investigativas en Educación, 3(11), 1-24. Recuperado de http://www.redalyc.org/ pdf/447/44722178014.pdf

Hernández, S.; Fernández, R. y Baptista, L. (2010). Metodología de la investigación. México: McGraw-Hill Interamericana. Recuperado de https://www.esup.edu.pe/descargas/dep_investigacion/Metodologia\%20de $\% 201 \mathrm{a} \% 20$ investigaci\%C3\%B3n $\% 20$ 5ta\%20Edici $\%$ C3\%B3n.pdf 
Jaén, L. (2011). Informe final del proceso de autoevaluación de la sección de archivística. San José: Universidad de Costa Rica.

Luengo, J.; Luzón, A. y Torres, M. (2008). Las reformas educativas basadas en el enfoque por competencias: una visión comparada. Revista de currículum y formación del profesorado, 12(3), 1-10. Recuperado de https://www.ugr.es/ recfpro/rev123ed.pdf

Monje, C. (2011). Metodología de la investigación cuantitativa y cualitativa: Guía Didáctica. Recuperado de https:/www.uv.mx/ rmipe/files/2017/02/Guia-didactica-metodologia-de-la-investigacion.pdf

Posada, R. (2004). Formación superior basada en competencias, interdisciplinariedad y trabajo autónomo del estudiante. Revista Iberoamericana de Educación, 35(1), 1-33. Recuperado de http:// www.ugr.es/ ugr_unt/Material\%20M\%F3dulo\%205/competencias_univ.pdf

Tobón, S.; Pimienta, H. y García, J. (2010). Secuencias didácticas: aprendizaje y evaluación de competencias. México: Pearson. Recuperado de http://files.ctezona141.webnode.mx/200000004-8ed038fca3/secuencias-didacticastobon-120521222400-phpapp02.pdf 